\section{CONTRIBUIÇĀO AO ESTUDO DOS RAMOS ARTERIAIS E DAS RAIZES VENOSAS, HILARES E EXTRAHIILARES, EM RINS DE CAPRINOS (Capra hircus)}

\author{
VICENTE DE PAULO JOHNSCHER GUARENTI \\ Professor Titular \\ Universidade Federal de Pelotas
}

ANTONIO FERNANDES FILHO

Professor Adjunto

Faculdade de Medicina Veterinária e

Zootecnia da USP
GUARENTI, V.P.J. \& FERNANDES FILHO, A. Contribuição ao estudo dos ramos arteriais e das raizes venosas, hilares e extrahilares, em rins de caprinos (Capra hircus). Rev.Fac.Med.vet. Zootec.Univ.S.Paulo, 22(2): 115-33, 1985.

RESUMO: Foram examinados 30 pares de rins de caprinos, sem raça definida, machos, adultos, oriundos de várias regiōes do estado de São Paulo. Os aludidos órgãos, retirados convenientemente da cavidade abdominal, sempre unidos aos tratos da aorta e da veia cava caudal, foram dissecados, depois de fixados em solução aquosa de formol a $10,0 \%$ durante 48 horas. Os resultados obtidos permitem chegar ìs seguintes conclusōes: 1) na região hilar do rim dircito identificam-se de cinco a quatorze e, no esquerdo, de seis a dezesseis ramos da A. renal; 2) na zona extrahilar do rim direito encontra-se de um a três e, no esquerdo, de um a quatro ramos de A. renal; 3) na regiāo hilar do rim direito contam-se de três a oito e, no esquerdo, de três a sete raízes venosas; 4) na zona extrahilar do rim direito verifica-se de uma a três e, no esquerdo, de uma a quatro raízes venosas; 5) a análise estatística (coeficiente de correlação de Pearson) mostra correlação positiva entre o número de ramos arteriais e raizes venosas, localizados no hilo, seja para o rim direito, seja para o rim esquerdo.

UNITERMOS: Anatomia, caprinos ${ }^{+}$; Rins $^{+}$; Artérias $^{+}$; Veias $^{+}$

\section{INTRODUÇÃO}

As informações obtidas nos compêndios dedicados à anatomia dos animais domésticos e mesmo em publicaç̃̃es especializadas, são muito escassas no tocante ao estudo dos vasos renais em pequenos ruminantes, como já observou PEDUTI NETO ${ }^{3}$ (1970), ao cuidar do focado assunto em carneiros, fato que se repete ao buscarmos dados semelhan tes para caprinos. Tal condição levou-nos a investigar, nestes animais, o número, a disposição e as regiōes de entrada e saida, respectivamente, dos ramos da artéria renal e raízes da veia renal, implicados apenas na vascularização do rim, com o objetivo de fornecer elementos de interesse ao conhecimento mais pormenorizado das aludidas entidades anatômicas, na Capra hircus, a fim de, não só estabelecer possíveis diferenças entre esta espécie e o Ovis aries, como também obter subsídios para o desenvolvimento da Anatomia Comparativa, visto que as referidas espécies, na maioria dos tratados, são apresentadas sob descriç̃̃o única.

\section{LITERATURA}

$\mathrm{Na}$ literatura que tivemos a oportunidade de compulsar, notamos a ausência de publicações especializadas referentes ao número e disposição dos vasos renais em caprinos. Porém, se levarmos em conta os pequenos ruminantes, encontramos informações atinentes aos ovinos, sobre o focado assunto, na pesquisa de PEDUTI NETO 3 (1970), realizada com metodologia semelhante à que agora empregamos.

Assim este A. assinala que o rim direito destes animais apresenta oito $(20,0 \%)$, sete $(16,7 \%)$, nove $(16,7 \%)$, dez $(16,7 \%)$, seis $(13,4 \%)$, onze $(3,3 \%)$, doze $(3,3 \%)$, quatorze $(3,3 \%)$ e o esquerdo, sete $(33,3 \%)$, oito $(16,7 \%)$, nove $(13,4 \%)$, seis $(10,0 \%)$, quatro $(6,7 \%)$, dez $(6,7 \%)$, cinco $(3,3 \%)$, onze $(3,3 \%)$, doze $(3,3 \%)$ e treze $(3,3 \%)$ ramos da artéria renal, que alcançam a regiāo hilar, ocupando disposiçāo predominantemente periférica, 27 vezes $(90,0 \%)$ à direita e 28 vezes $(93,3 \%)$ dे esquerda, prevalentemente central, 2 vezes $(6,7 \%)$ de cada lado, periférica e central equivalente, apenas 1 vez $(3,3 \%)$, do lado direito. Quanto às raizes das veias renais, registra, junto ao hilo, no rim direito, quatro $(33,3 \%)$, duas $(23,3 \%)$, três $(16,7 \%)$, cinco $(13,3 \%)$, seis $(6,7 \%)$, sete $(6,7 \%)$ e no esquerdo, quatro $(40,0 \%)$, dois $(20,0 \%)$, três $(16,7 \%)$, cinco $(16,7 \%)$, seis $(3,3 \%)$, que aparecem na regiāo hilar, em situaçāo exclusivamente periférica, 19 vezes $(63,3 \%)$ à direita e 13 vezes $(43,3 \%)$ à esquerda, preponderantemente periférica, na mesma ordem, 8 vezes $(26,7 \%)$ e 12 vezes $(40,0 \%)$, periférica e central equivalentes, 3 vezes $(10,0 \%)$ do lado direito e 4 vezes $(13,4 \%)$ do esquerdo e com prevalência, central, 1 vez $(3,3 \%)$ do lado esquerdo. Confrontando os rins direito e esquerdo. evidencia, aiıda, o A. igual número de ramos arıulais, somente 5 vezes $(16,7 \%)$ e, de raizes venosas 15 vezes $(50,0 \%)$, sendo que destas, $5(16,7 \%)$ mostraram mesma disposiçāo, relativamente às linhas craniocaudal e dorsoventral e aos qua- 
drantes craniodorsal, cranioventral, caudodorsal e caudoventral. Estatisticamente, demonstrou, também, correlação positiva entre o número de ramos arteriais e raízes venosas encontradas no hilo, seja para o rim direito, seja para o esquerdo, bem como entre os ramos arteriais ou raízes venosas, separadamente, quando comparados os dois rins do mesmo animal.

Já, no tocante aos compêndios dedicados à Anatomia Veterinária, cumpre-nos ressaltar que mesmo aqueles que consideram isoladamente os ruminantes (MONTANÉ \& BOURDELLE $^{2}$, 1917; MARTIN \& SCHAUDER ${ }^{1}$, 1938), nada informam sobre o número e disposição dos elementos vasculares do hilo renal nos caprinos, fazendo na maioria das vezes, alusão apenas aos equinos, sem contudo esclarecerem as diferenças existentes em relação aos pequenos ruminantes, ou a eles se reportam de maneira apenas genérica, não fornecendo a nosso ver dados que permitam um eventual confronto com os resultados agora obtidos.

\section{MATERIAL E MÉTODO}

Para a realização desta pesquisa utilizamos 30 pares de rins, pertencentes a caprinos, sem raça definida, machos, adultos, oriundos de várias regióes do estado de São Paulo.

Os aludidos órgãos, retirados convenientemente da cavidade abdominal, sempre unidos aos tratos da aorta e da veia cava caudal, foram dissecados, depois de fixados mediante injeção de solução aquosa de formol a $10,0 \%$ nas artérias renais, conservando-os imersos em igual solução durante no mínimo 48 horas. Das peças, uma vez preparadas, colhemos desenhos esquemáticos para ulterior análise e documentação.

Para a descrição dos resultados, consideramos as regioes hilar e extrahilar, demarcando nelas, quadrantes, mediante o auxilio de duas linhas imaginárias, perpendiculares entre si, traçadas de polo a polo e no sentido dorsoventral, cuja intersecção coincide com o centro de orifício da secçđo transversa justarenal do ureter.

Assim, obtivemos quadrantes que receberam as seguintes denominaçōes: craniodorsal, cranioventral, caudodorsal e caudoventral, os quais foram tomados de base para o registro do número e locais de entrada e saída, respectivamente, dos ramos arteriais e das raizes venosas, conforme apresentamos nos esquemas realizados que representam os pares de rins, submetidos a giro de $90^{\circ}$, obedecendo o sentido medioventral (Fig. 1 a 30 correspondentes às observaçōes de 1 a 30 ).

\section{RESULTADOS}

Com base nas dissecaçðes dos 30 pares de rins de ca. prinos, apresentamos os resultados, correspondentes ao número, disposição e às regiðes de entrada e saída, respectivamente, dos ramos da artéria renal e das raizes da veia renal (Fig. 1 a 30), obedecendo os quadrantes e linhas, previamente traçados para os rins direito e esquerdo, conforme exposto nos Quad. 1,2,3,4.

\section{COMENTÁRIOS}

Como tivemos oportunidade de ressaltar, anteriormente, quanto às informaçőes atinentes aos elementos vasculares renais em caprinos, nada encontramos nas publicações especializadas, repetindo-se o mesmo fato no que tange aos livros de texto. Na realidade, a maioria dos tratadistas toma o equino como padrão em suas descriçōes, deixando de registrar, em relação ao focado assunto, as possiveis diferenças existentes quando considera comparativamente a $\mathrm{A}$ natomia dos Animais Domésticos. Cabe mesmo destacar que MONTANÉ \& BOURDELLE ${ }^{2}$ e MARTIN \& SCHAUDER $^{1}$, apesar de focalizarem em especial os ruminantes, não oferecem em seus compêndios, dados pertinentes às variaçōes anatômicas, agora estudadas em caprinos. De outra parte, entendemos válido o cotejo dos resultados agora obtidos em caprinos, com os verificados por PEDUTI $\mathrm{NETO}^{3}$ em ovinos, por utlizar este A. a mesma metodologia por nós empregada, o que proporcionará conhecimento de possiveis diferenças entre esses pequenos ruminantes, cuja anatomia é, frequentemente, cuidada em conjunto.

Assim, com relação ao número de ramos arteriais: PEDUTI NETO ${ }^{3}$, em ovinos, registra junto ao hilo, de três a catorze e de quatro a treze ramos, respectivamente, para 0 rim direito e esquerdo, enquanto encontramos valores que muito dele se aproxima, isto é, cinco a catorze e seis a de. zesseis obedecendo a mesma ordem de citaç̃o. No tocante às raizes da veia renal, vistas na região hilar, aquele $\mathrm{A}$. a no ta de duas a sete para ambos os rins, arranjo este verificado em caprinos, com números quase semelhantes, vale dizer, de três a oito à direita e de três a sete à esquerda.

Ainda, se levarmos em conta o número de ramos da artéria renal e o das raízes da veia renal a penetrarem e a e mergirem, respectivamente, da regiāo extrahilar verificamos para os primeiros, de um a très à direita e, de um a quatro á esquerda, contando-se de uma a très à direita e, de uma c quatro, à esquerda, relativamente às últimas. Cabe destacas que estes achados não possibilitam confronto com os de PEDUTI NETO ${ }^{3}$, pois, este A. ñ̃o faz menç̃o aos elemen tos vasculares da região extrahilar.

Já, quanto à disposição dos elementos vasculares, ago ra estudados, devemos esclarecer que, tanto os amos arte riais como as raizes venosas, acham-se sempre localizadas perifericamente; entretanto PEDUTI NETO ${ }^{3}$, referindo.se 
QUADRO 1 - Número de ramos arteriais hilares e extrahilares da artéria renal direita de caprinos. São Paulo, 1985.

\begin{tabular}{|c|c|c|c|c|c|c|c|c|}
\hline \multirow{4}{*}{ Dos. } & \multicolumn{7}{|c|}{ Localizaçà o } & \multirow{4}{*}{ Total } \\
\hline & \multicolumn{5}{|c|}{ Regiãa Hilar } & \multirow{3}{*}{\multicolumn{2}{|c|}{$\begin{array}{c}\text { Região } \\
\text { Extrahilar }\end{array}$}} & \\
\hline & \multicolumn{4}{|c|}{ Quadrantes } & \multirow{2}{*}{$\begin{array}{c}\text { Limites dos } \\
\text { Quadrantes }\end{array}$} & & & \\
\hline & CRV & CRD & CAV & CAD & & & & \\
\hline 1 & 3 & 3 & 2 & 3 & CRV - CAV - 1 & & - & 12 \\
\hline 2 & 4 & 4 & 3 & 3 & - & & - & 14 \\
\hline 3 & 2 & 2 & 3 & 1 & CRV - CRD - 1 & CRV & -1 & 10 \\
\hline 4 & 2 & 1 & 2 & 1 & CRV - CRD - 1 & & - & 7 \\
\hline 5 & 3 & 2 & 3 & 2 & - & & - & 10 \\
\hline 6 & 2 & 3 & 2 & 2 & - & & - & 9 \\
\hline 7 & 3 & 3 & 2 & 2 & - & & - & 10 \\
\hline 8 & 2 & 1 & 1 & 1 & $\begin{array}{l}\text { CRV - CRD - } 1 \\
\text { CRD - CAD - } 1\end{array}$ & & - & 7 \\
\hline 9 & 3 & 3 & 2 & 1 & - & & - & 9 \\
\hline 10 & 2 & 1 & 3 & 1 & $\begin{array}{l}\text { CRV - CRD - } 1 \\
\text { CRD - CAD - } 1\end{array}$ & & - & 9 \\
\hline 11 & 3 & 2 & 2 & 1 & - & CRV & -1 & 9 \\
\hline 12 & 4 & 3 & 3 & 1 & - & & - & 11 \\
\hline 13 & 1 & 3 & 2 & 1 & CRV - CAV - 1 & & - & 8 \\
\hline 14 & 3 & 3 & 2 & 1 & - & & - & 9 \\
\hline 15 & 5 & 4 & 2 & 3 & - & & - & 14 \\
\hline 16 & 2 & 3 & 2 & 3 & - & & - & 10 \\
\hline 17 & 1 & 2 & 1 & 3 & CRV - CAV - I & & - & 8 \\
\hline 18 & 2 & 1 & - & 2 & - & CRV & -2 & 7 \\
\hline 19 & 2 & 2 & 2 & 2 & $\begin{array}{l}\text { CRV - CAV - I } \\
\text { CRD - CAD - } 1\end{array}$ & & & 10 \\
\hline$\cdot 20$ & 2 & $2^{5}$ & 1 & 1 & $\begin{array}{l}\text { CRD - CAD - I } \\
\text { CAU - CAD - I }\end{array}$ & $\begin{array}{l}\text { CRV } \\
\text { CRD }\end{array}$ & $\begin{array}{l}-1 \\
-2\end{array}$ & 11 \\
\hline 21 & 2 & 3 & 2 & 2 & $\begin{array}{l}\text { CRV - CRD - I } \\
\text { CRV - CAV - }\end{array}$ & & & 11 \\
\hline 22 & 4 & 3 & 2 & 1 & - & & - & 10 \\
\hline 23 & 2 & 4 & 3 & $I$ & - & & - & 10 \\
\hline 24 & 2 & 4 & 2 & 1 & - & $\begin{array}{l}\text { CRV } \\
\text { CRD }\end{array}$ & $\begin{array}{l}-1 \\
-1\end{array}$ & 11 \\
\hline 25 & 1 & 2 & 2 & 2 & - & & - & 7 \\
\hline 26 & 3 & 3 & 4 & 4 & - & CRV & -1 & 15 \\
\hline 27 & 2 & 3 & 3 & 2 & - & & - & 10 \\
\hline 28 & 3 & 3 & 2 & 3 & $C R V-C A V-1$ & & - & 12 \\
\hline 29 & 2 & 2 & 3 & 2 & - & & - & 9 \\
\hline 30 & 4 & 3 & 1 & 2 & - & & - & 10 \\
\hline
\end{tabular}


(Capra hircus)

QUADRO 2 - Número de raízes hilares e extrahilares da veia renal direita de caprinos. São Paulo, 1985.

\begin{tabular}{|c|c|c|c|c|c|c|c|c|}
\hline \multirow{4}{*}{ Obs. } & \multicolumn{7}{|c|}{ Localizaça } & \multirow{4}{*}{ Total } \\
\hline & \multicolumn{5}{|c|}{ Regiad HilaI } & \multirow{3}{*}{\multicolumn{2}{|c|}{$\begin{array}{c}\text { Regiãa } \\
\text { Extrahilar }\end{array}$}} & \\
\hline & \multicolumn{4}{|c|}{ Quadrantes } & \multirow{2}{*}{$\begin{array}{c}\text { Limites dos } \\
\text { Quadrantes }\end{array}$} & & & \\
\hline & CRV & CRD & CAV & CAD & & & & \\
\hline 1 & 3 & 2 & 2 & 1 & - & & - & 8 \\
\hline$\frac{1}{2}$ & 2 & 2 & 2 & 1 & - & & - & 7 \\
\hline 3 & 2 & 2 & 1 & - & $C R D-C A D-1$ & CRY & -1 & 7 \\
\hline 4 & - & 1 & 2 & 1 & CRV - CRD - I & & - & 5 \\
\hline 5 & - & 1 & 1 & 1 & CRV - CAV - I & & & \\
\hline & & & & & CRD - CAD - 1 & & - & 5 \\
\hline 6 & 2 & 1 & 1 & - & - & & - & 4 \\
\hline 7 & 1 & 1 & 2 & 1 & CRV - CRD - I & & - & 6 \\
\hline 8 & 1 & - & 1 & 1 & - & & - & 3 \\
\hline 9 & - & 1 & $\bar{l}$ & $\overline{1}$ & CRV - CAV - I & & - & 4 \\
\hline 10 & 2 & 2 & 1 & - & - & & - & 5 \\
\hline 11 & 1 & 1 & $i$ & - & CRV - CAV - I & & - & 4 \\
\hline 12 & 1 & 1 & 1 & 1 & CRV - CRD - I & & - & 5 \\
\hline 13 & 2 & - & 2 & - & CRD - CAD - I & & - & 5 \\
\hline 14 & 1 & 2 & 1 & - & - & & - & 4 \\
\hline 15 & 1 & - & 2 & - & CRV - CRD - I & & & \\
\hline & & & & & CRD - CAD - I & & - & 5 \\
\hline 16 & 1 & - & 1 & - & CRD - CAD - 1 & & - & 3 \\
\hline 17 & I & - & 2 & - & CRD - CAD - 1 & & - & 4 \\
\hline 18 & 1 & 1 & 1 & 2 & - & CRV & -2 & 7 \\
\hline 19 & - & 1 & 2 & 1 & CRV - CRD - 1 & & - & 5 \\
\hline 20 & 1 & 2 & 2 & 1 & CRV - CAV - I & CRV & -1 & \\
\hline & & & & & & CRD & -2 & 10 \\
\hline 21 & 1 & 2 & 1 & 1 & CRV - CAV - I & & & \\
\hline & & & & & CAU - CAD - 1 & & - & 7 \\
\hline 22 & 2 & 1 & 1 & 1 & - & & - & 5 \\
\hline 23 & 2 & 1 & 1 & 1 & - & & - & 5 \\
\hline 24 & 2 & 1 & 2 & - & - & & - & 5 \\
\hline 25 & 1 & 2 & 1 & - & - & & - & 4 \\
\hline 26 & 2 & 1 & 2 & 1 & - & & - & 6 \\
\hline 27 & 2 & 1 & 2 & - & - & & - & 5 \\
\hline 28 & 1 & 2 & 2 & 1 & - & & - & 6 \\
\hline 29 & 2 & 1 & - & 1 & - & & - & 4 \\
\hline 30 & 1 & 1 & 1 & 1 & CRV - CRD - 1 & & - & 5 \\
\hline
\end{tabular}

Rev.Fac.Med.vet.Zootec.Univ.S.Paulo, 22(2): 115-33, 1985. 
QUADRO 3 - Número de ramos arteriais hilares e extrahilares da artéria renal esquerda de caprinos. Paulo, 1985.

\begin{tabular}{|c|c|c|c|c|c|c|c|c|}
\hline \multirow{4}{*}{ Obs. } & \multicolumn{7}{|c|}{ Lócalizaçã o } & \multirow{4}{*}{ Tatal } \\
\hline & \multicolumn{5}{|c|}{ Região Hilar } & \multirow{3}{*}{\multicolumn{2}{|c|}{$\begin{array}{c}\text { Região } \\
\text { Extranilar }\end{array}$}} & \\
\hline & \multicolumn{4}{|c|}{ Quadrantes } & \multirow{2}{*}{$\begin{array}{l}\text { Limites das } \\
\text { Luadrantes }\end{array}$} & & & \\
\hline & CRV & CRD & CAV & CAD & & & & \\
\hline 1 & 3 & 2 & 2 & 2 & _ & & - & 9 \\
\hline $\begin{array}{l}1 \\
2\end{array}$ & 4 & 2 & 4 & 4 & - & CRV & -1 & 15 \\
\hline 3 & 4 & 3 & 4 & 3 & - & CRV & -1 & 15 \\
\hline 4 & 1 & 4 & 3 & 1 & - & & - & 9 \\
\hline 5 & 2 & 3 & 2 & 2 & - & & - & 9 \\
\hline 6 & 2 & 3 & 3 & 2 & - & & - & 10 \\
\hline 7 & 3 & 3 & 2 & 2 & - & & - & 10 \\
\hline 8 & 4 & 3 & 2 & 2 & - & & - & 11 \\
\hline 9 & 3 & 3 & 3 & 2 & - & & - & 11 \\
\hline 10 & 3 & 3 & 3 & 2 & - & & - & 11 \\
\hline 11 & 4 & 3 & 1 & 1 & - & & - & 9 \\
\hline 12 & 3 & 3 & 1 & 2 & CRV - CAV - 1 & & - & 10 \\
\hline 13 & 3 & 2 & 2 & 1 & $C R D-C A D-1$ & & - & 9 \\
\hline \multirow[t]{2}{*}{14} & 2 & 2 & 1 & 1 & CRV - CAV - I & CRV & -1 & \\
\hline & & & & & & CRD & -1 & 9 \\
\hline 15 & 4 & 4 & 6 & 2 & - & & - & 16 \\
\hline 16 & 4 & 3 & 2 & 2 & $C A D-C R D-1$ & & - & 12 \\
\hline 17 & 4 & 3 & 2 & 2 & CAD - CRD - I & & - & 12 \\
\hline \multirow[t]{2}{*}{18} & 2 & 2 & 1 & 1 & - & CRV & -3 & \\
\hline & & & & & & CRD & -1 & 10 \\
\hline 19 & 3 & 3 & 2 & 1 & CAV - CAD - 1 & & - & 10 \\
\hline 20 & 3 & 3 & 2 & 2 & - & CRD & -2 & 12 \\
\hline \multirow[t]{2}{*}{21} & 3 & 3 & 1 & 2 & CFY - CAU - I & & - & \\
\hline & & & & & $C A D-C R D-1$ & CRV & -1 & 12 \\
\hline 22 & 3 & 3 & 3 & 2 & - & & - & 11 \\
\hline 23 & 2 & 3 & 4 & 3 & - & CRD & -1 & 13 \\
\hline 24 & 3 & 2 & 3 & 2 & - & & - & 10 \\
\hline 25 & 3 & 4 & 2 & 2 & - & & - & 11 \\
\hline 26 & 2 & 3 & 1 & 1 & - & & - & 7 \\
\hline 27 & 5 & 2 & 2 & 2 & CRV - CRD - 1 & & - & 12 \\
\hline 28 & 2 & 4 & 2 & 2 & CRV - CAV - I & & - & 11 \\
\hline 29 & 3 & 3 & 2 & 2 & - & CRV & -1 & 11 \\
\hline 30 & 5 & 3 & 4 & 3 & - & & - & 15 \\
\hline
\end{tabular}


QUADRO 4 - Número de raízes hilares e extrahilares da veia renal esquerda de caprinos. São Paulo, 1985.

\begin{tabular}{|c|c|c|c|c|c|c|c|}
\hline \multirow{4}{*}{ obs. } & \multicolumn{6}{|c|}{ Localização } & \multirow{4}{*}{ Total } \\
\hline & \multicolumn{5}{|c|}{ Região Hilar } & \multirow{3}{*}{$\begin{array}{c}\text { Regiã } \\
\text { Extrahilar }\end{array}$} & \\
\hline & \multicolumn{4}{|c|}{ Quadrantes } & \multirow{2}{*}{$\begin{array}{l}\text { Limftes dos } \\
\text { Quadrantes }\end{array}$} & & \\
\hline & CRV & CRD & CAV & CAD & & & \\
\hline 1 & 1 & 1 & 1 & 2 & - & & 5 \\
\hline 2 & 2 & 2 & 2 & 1 & - & & 7 \\
\hline 3 & 2 & 1 & 2 & - & CRV - CRD - 1 & CRV - 1 & 7 \\
\hline 4 & 1 & 2 & 1 & 1 & - & & 5 \\
\hline 5 & 1 & - & 1 & - & $\begin{array}{l}C R V \text { - CRD - } 1 \\
C A D \text { - CRD - } 1\end{array}$ & & 4 \\
\hline 6 & 1 & 2 & 1 & - & - & & 4. \\
\hline 7 & - & 1 & 1 & 1 & $\begin{array}{l}\text { CRV - CRD - } 1 \\
\text { CRV - CAV - I }\end{array}$ & & 5 \\
\hline 8 & - & 1 & 1 & - & $\begin{array}{l}\text { CRV - CAV - I } \\
\text { CAD - CRD - I }\end{array}$ & & 4 \\
\hline 9 & 1 & 1 & 1 & 1 & - & & 4 \\
\hline 10 & 1 & 1 & 2 & 2 & - & & 6 \\
\hline 11 & 1 & - & 1 & - & $\begin{array}{l}\text { CRU - CAV - } 1 \\
\text { CAD - CRD - } 1\end{array}$ & & 4 \\
\hline 12 & 1 & 1 & 3 & 1 & CRV - CRD - I & & 7 \\
\hline 13 & 2 & 1 & 1 & 1 & CAD - CRD - I & & 6 \\
\hline 14 & 1 & 1 & - & 1 & $\begin{array}{l}\text { CRV - CAV - } 1 \\
\text { CAD - CRD - } 1\end{array}$ & & 5 \\
\hline 15 & 1 & 1 & 1 & 2 & - & & 5 \\
\hline 16 & 2 & 1 & 2 & - & CRV - CRD - 1 & & 6 \\
\hline 17 & - & - & 1 & - & $\begin{array}{l}C R V-C A V-1 \\
C A D-C R D-1\end{array}$ & & 3 \\
\hline 18 & 1 & 1 & 2 & 1 & CRV - CRD - I & $\begin{array}{l}\text { CRV }-3 \\
\text { CRD }-1\end{array}$ & 10 \\
\hline 19 & 2 & 2 & $1=$ & - & CRV - CAV - I & & 6 \\
\hline 20 & 2 & - & 2 & - & CAD - CRD - I & $\begin{array}{c}\text { CRV - I } \\
\text { CRU - CRD - I }\end{array}$ & 7 \\
\hline 21 & 1 & 1 & 2 & 2 & CRV - CRD - 1 & CRV - 1 & 8 \\
\hline 22 & 3 & - & 1 & 2 & - & & 6 \\
\hline 23 & 3 & 2 & 2 & - & - & & 7 \\
\hline 24 & 1 & 2 & 2 & 1 & & & 6 \\
\hline 25 & 1 & 2 & 2 & - & & & 5 \\
\hline 26 & 1 & 1 & 1 & - & CRV - CRD - 1 & & 4 \\
\hline 27 & 2 & $i$ & 1 & 1 & - & & 5 \\
\hline 28 & 1 & 2 & 2 & 1 & CRV - CRD - I & & 7 \\
\hline 29 & 1 & 1 & 1 & 1 & CRV - CRD - I & & 5 \\
\hline 30 & 1 & 2 & 2 & 1 & - & & 6 \\
\hline
\end{tabular}


Representações esquemáticas dos ramos arteriais e das raízes venosas das regiōes hilar (a) e extrahilar (b), nos rins direito (D) e esquerdo (E), de caprinos (Capra hircus), machos, considerando os órgãos submetidos a giro $90^{\circ}$, no sentido médio-ventral. (Fig. 1 a 30 corresponde às observações de 1 a 30).

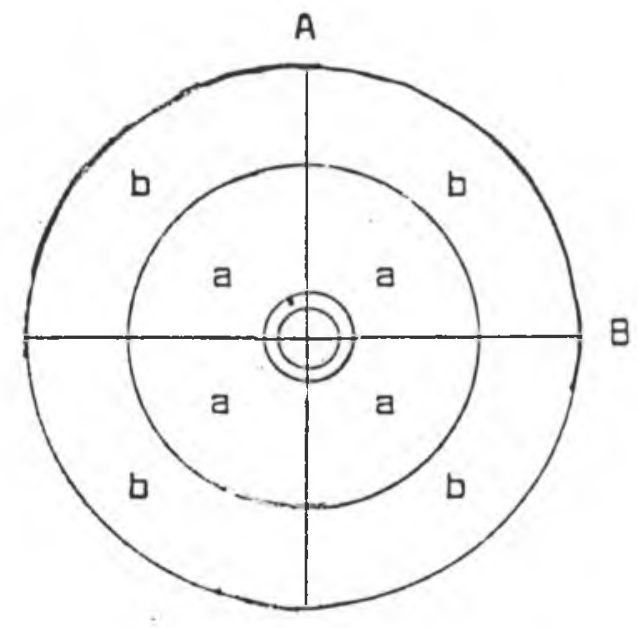

$$
\begin{aligned}
& \text { A - linha craniocaudal } \\
& \text { B - linha dorsoventral } \\
& \text { I - quadrante cranioventral } \\
& \text { III - quadrante caudodorsal } \\
& \text { IV - quadrante caudoventral } \\
& \text { - ramoter }
\end{aligned}
$$




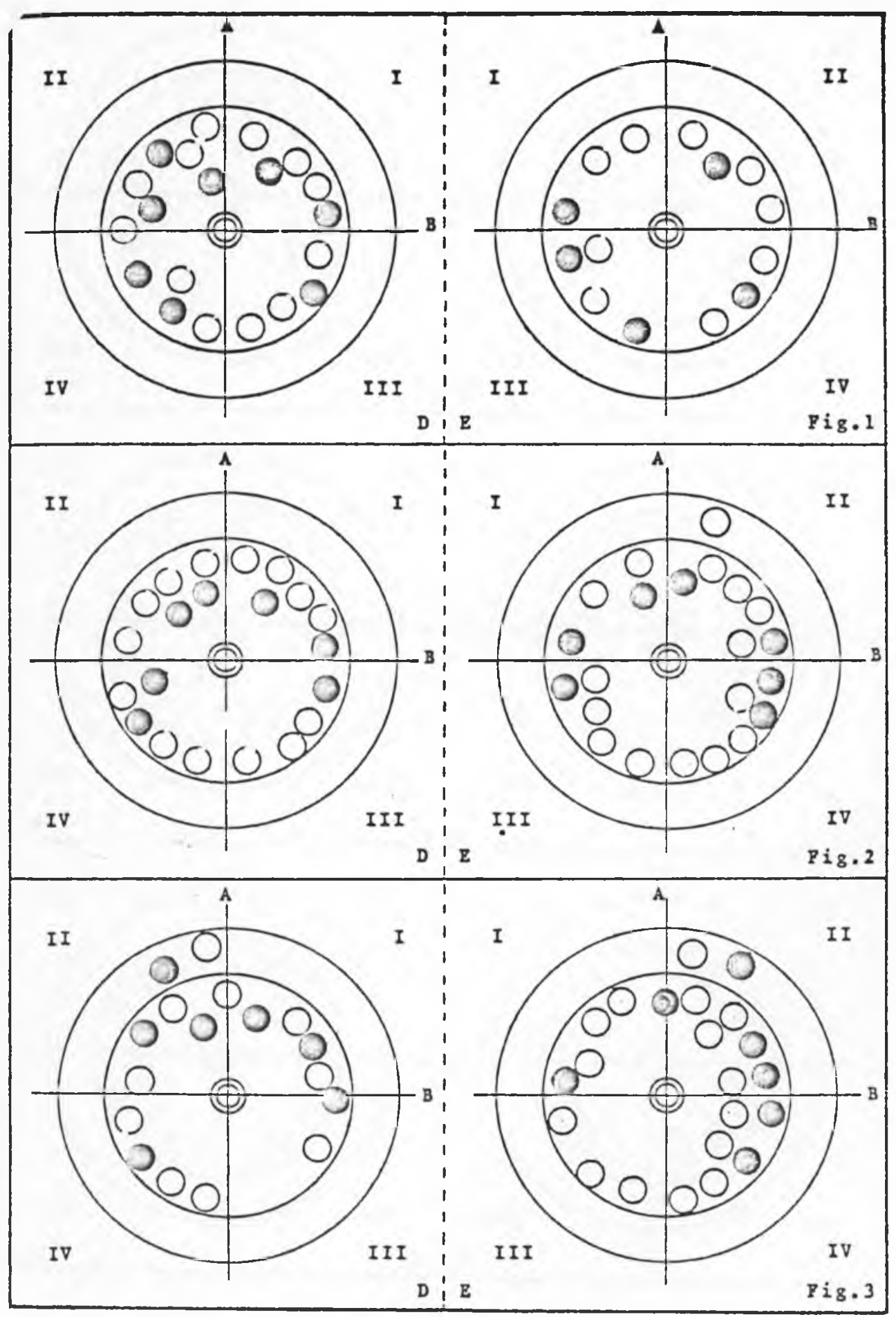

Rev.Fac.Med.vet.Zootec.Univ.S.Paulo, 22(2): 115-33, 1985. 


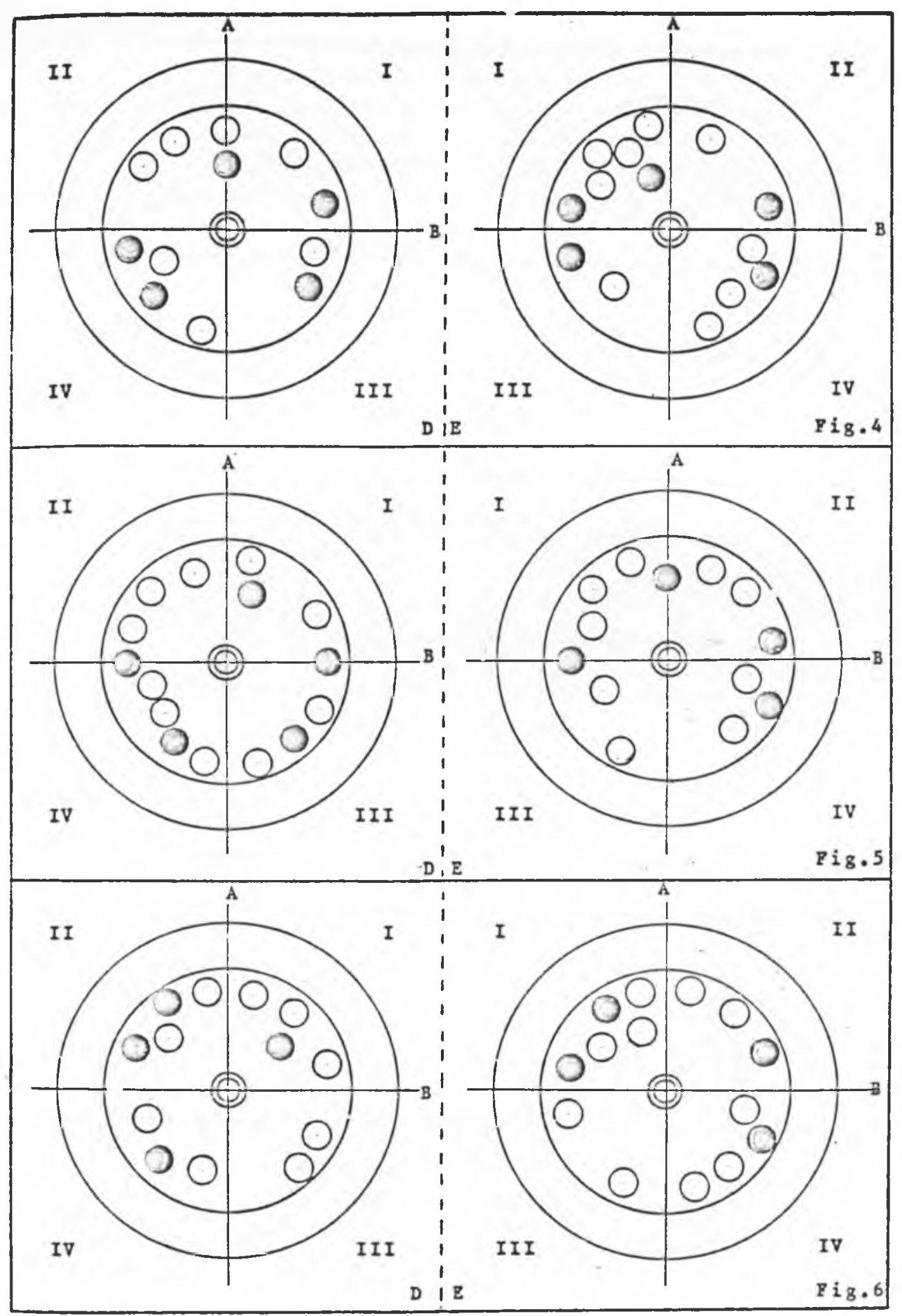




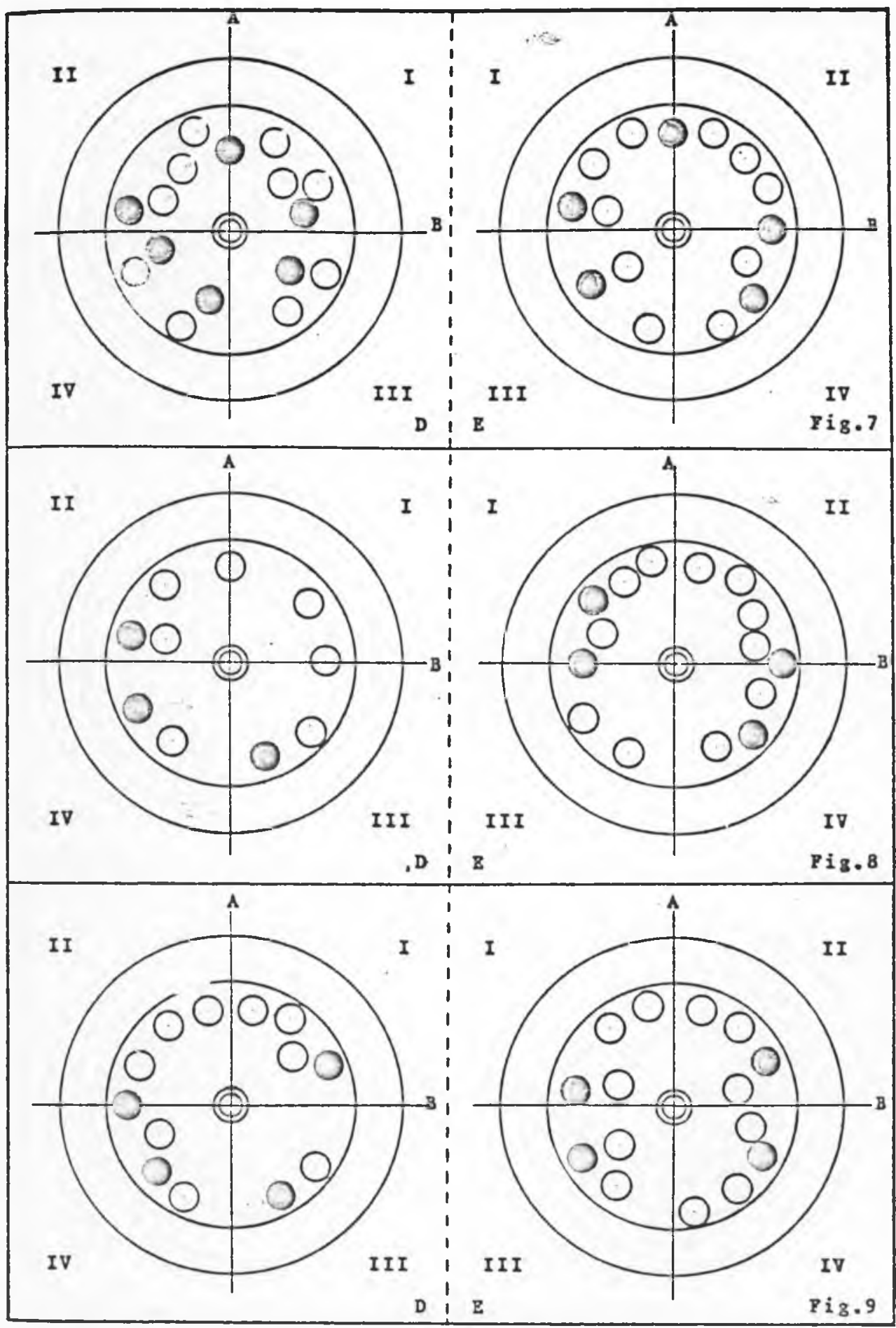

Rev.Fac.Med.vet.Zootec.Univ.S.Paulo, 22(2): 115-33, 1985. 


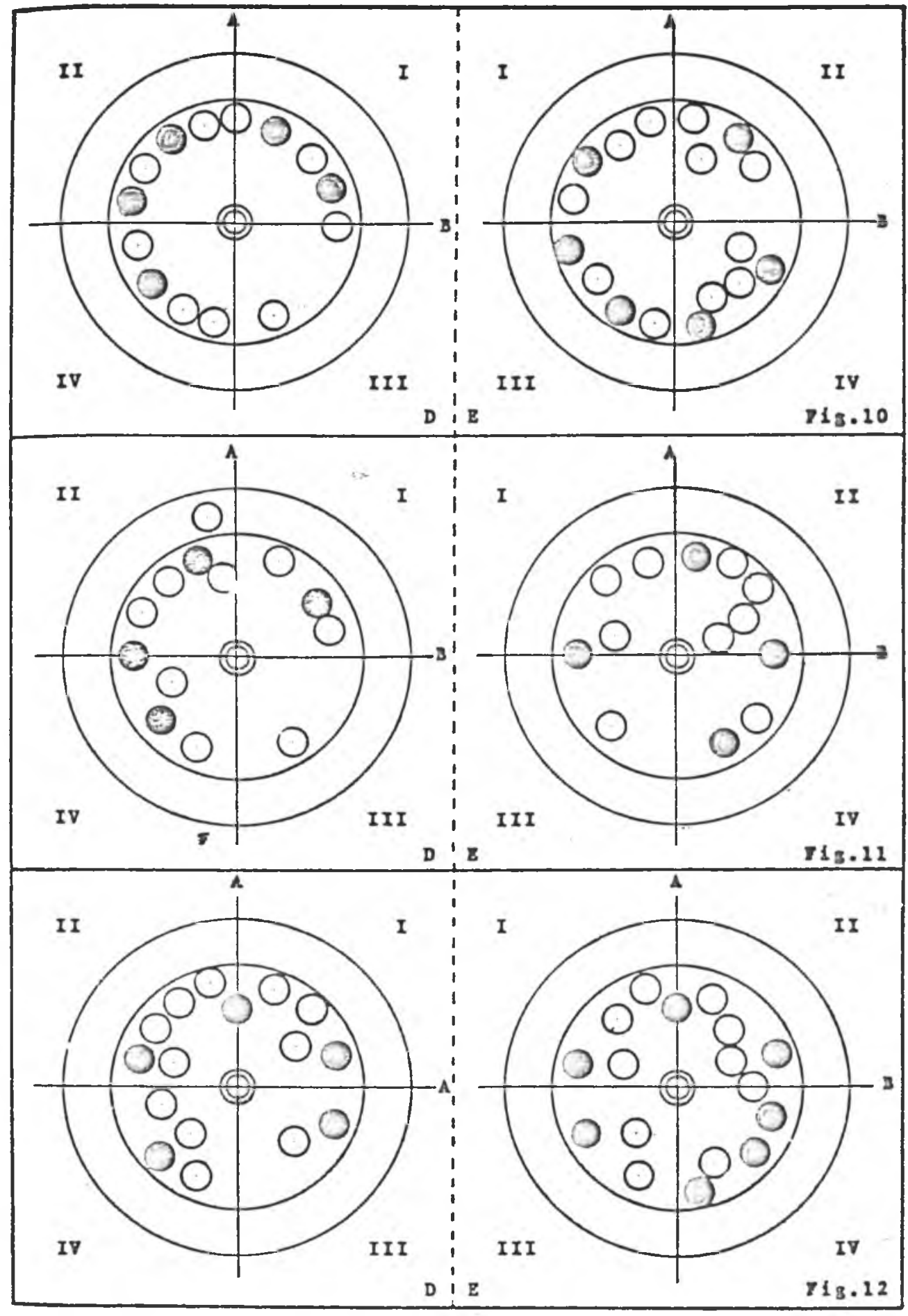

Rev.Fac.Med.vet.Zootec.Univ.S.Paulo, 22(2): 115-33, 1985. 


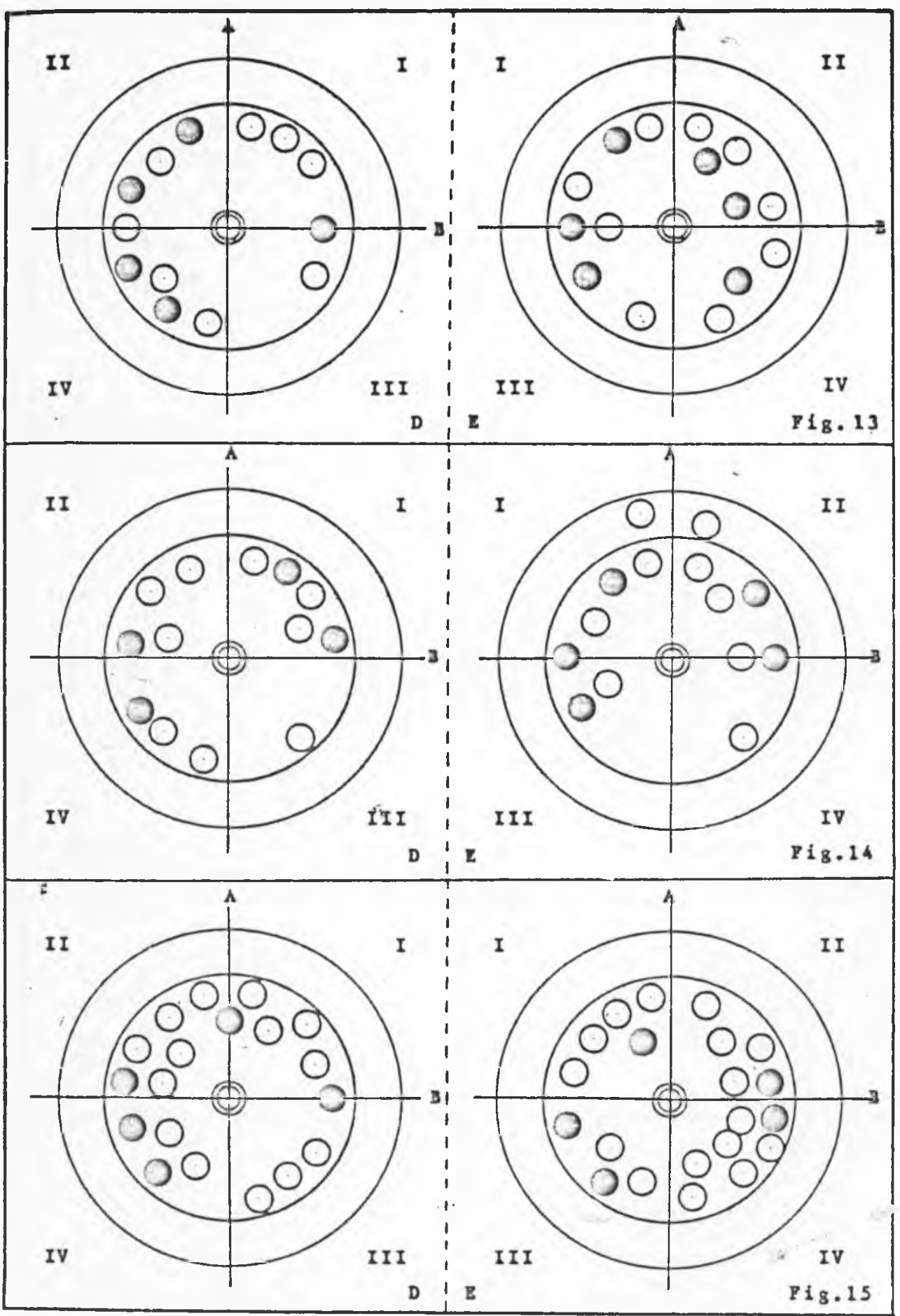

Rev.Fac.Med.vet.Zootec.Univ.S.Paulo, 22(2): 115-33, 1985. 


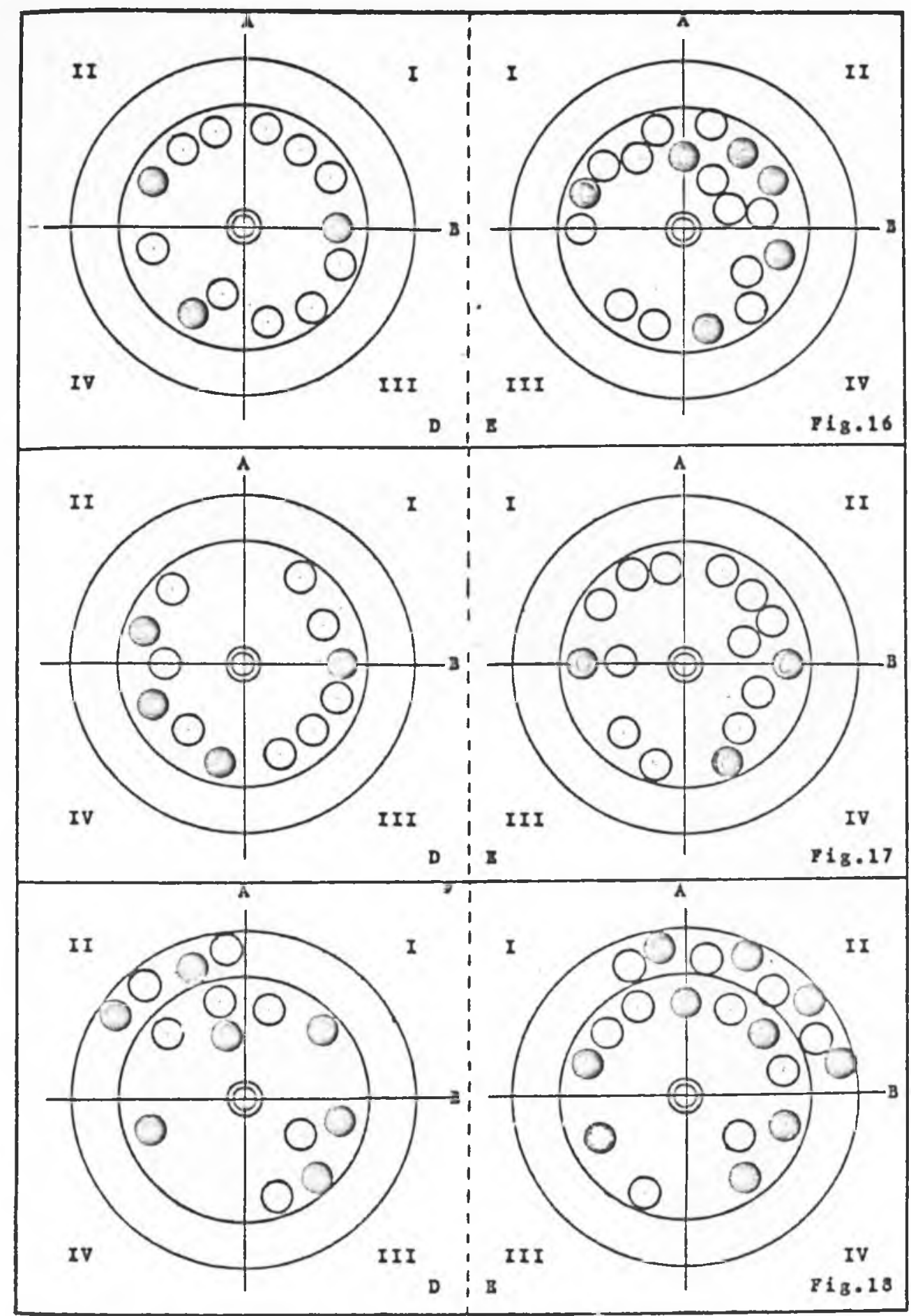

Rev.Fac.Med.vet.Zootec.Univ.S.Paulo, 22(2): 115-33, 1985. 


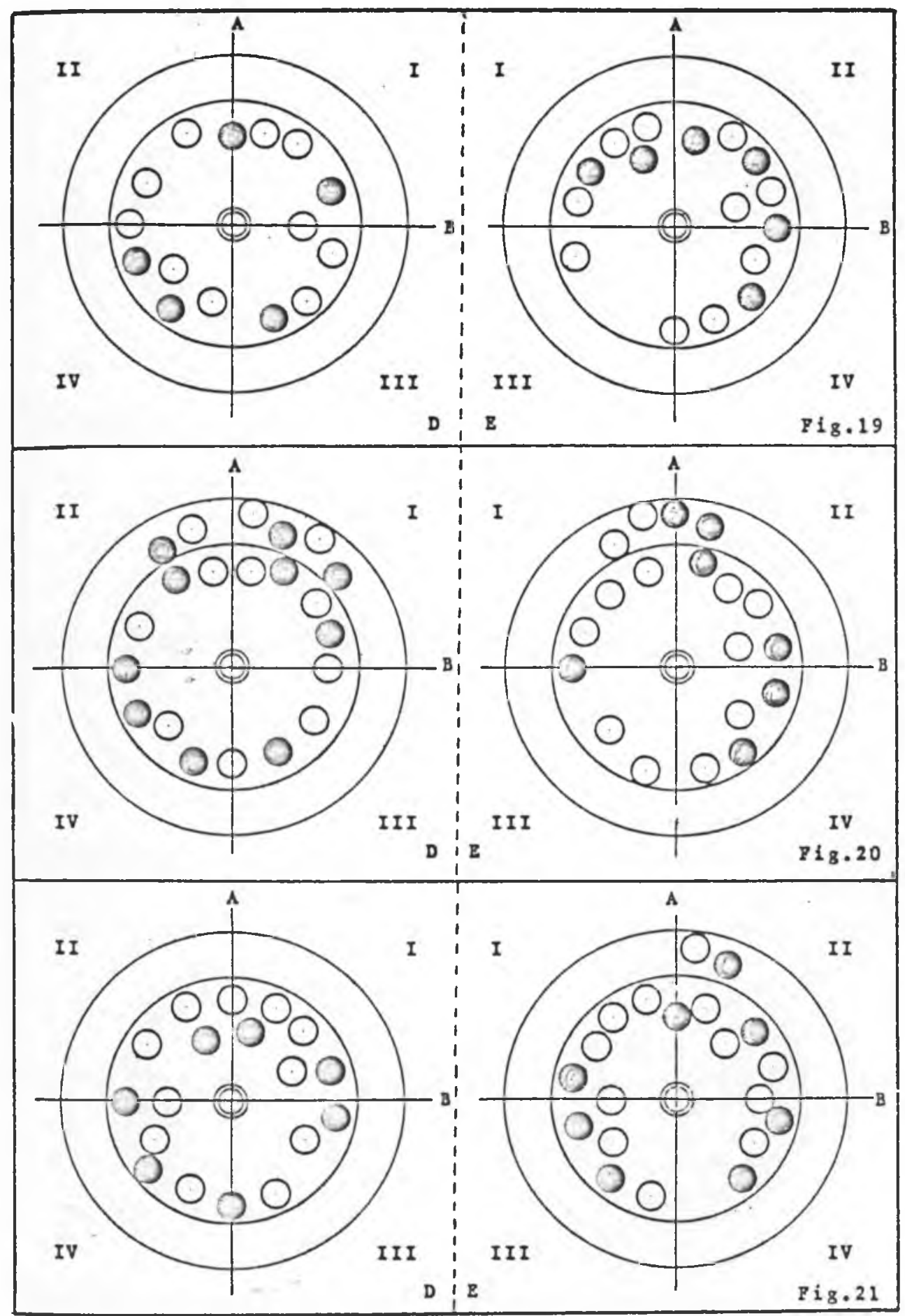

Rev.Fac.Med.vet.Zootec.Univ.S.Paulo, 22(2): 115-33, 1985. 


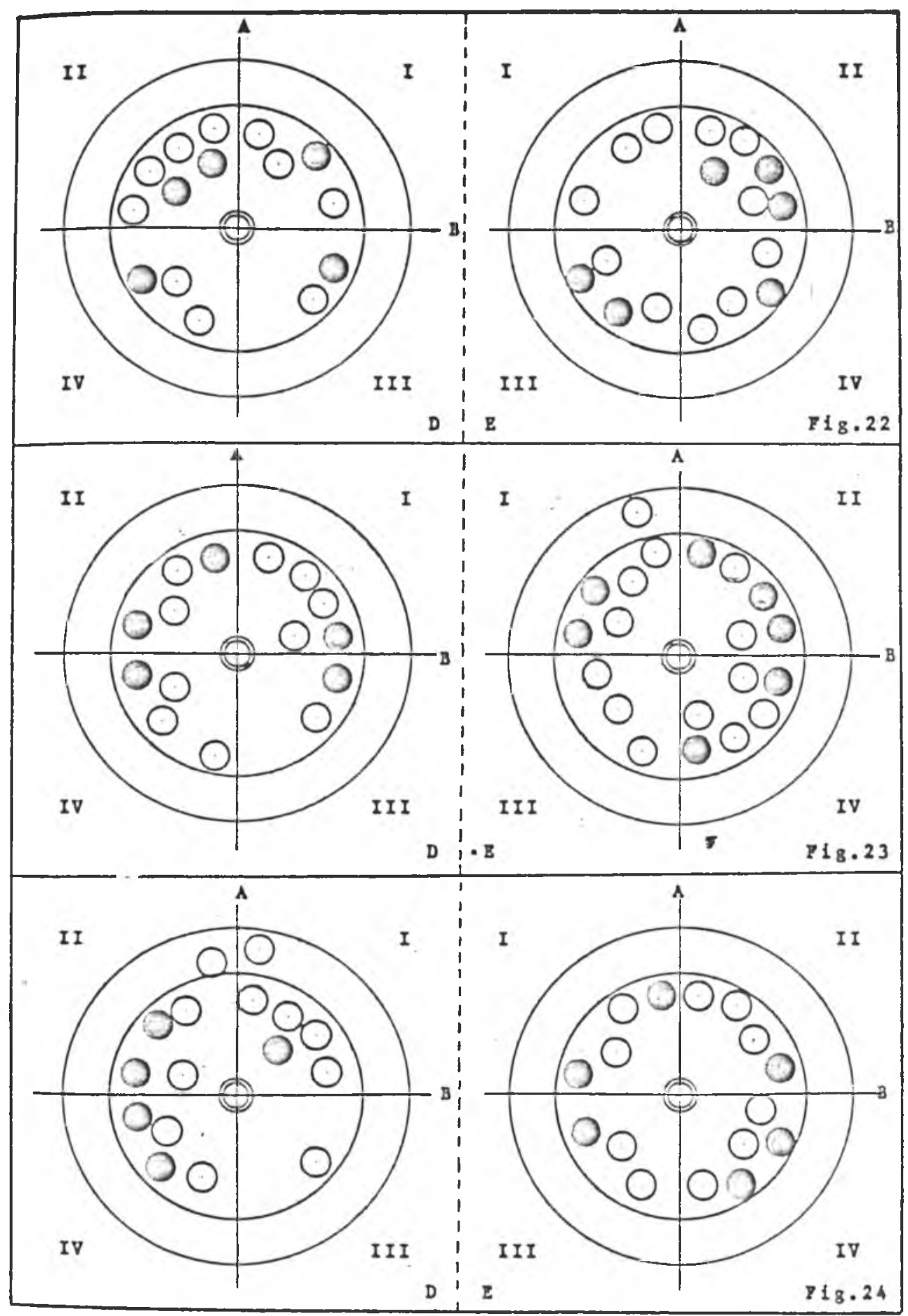




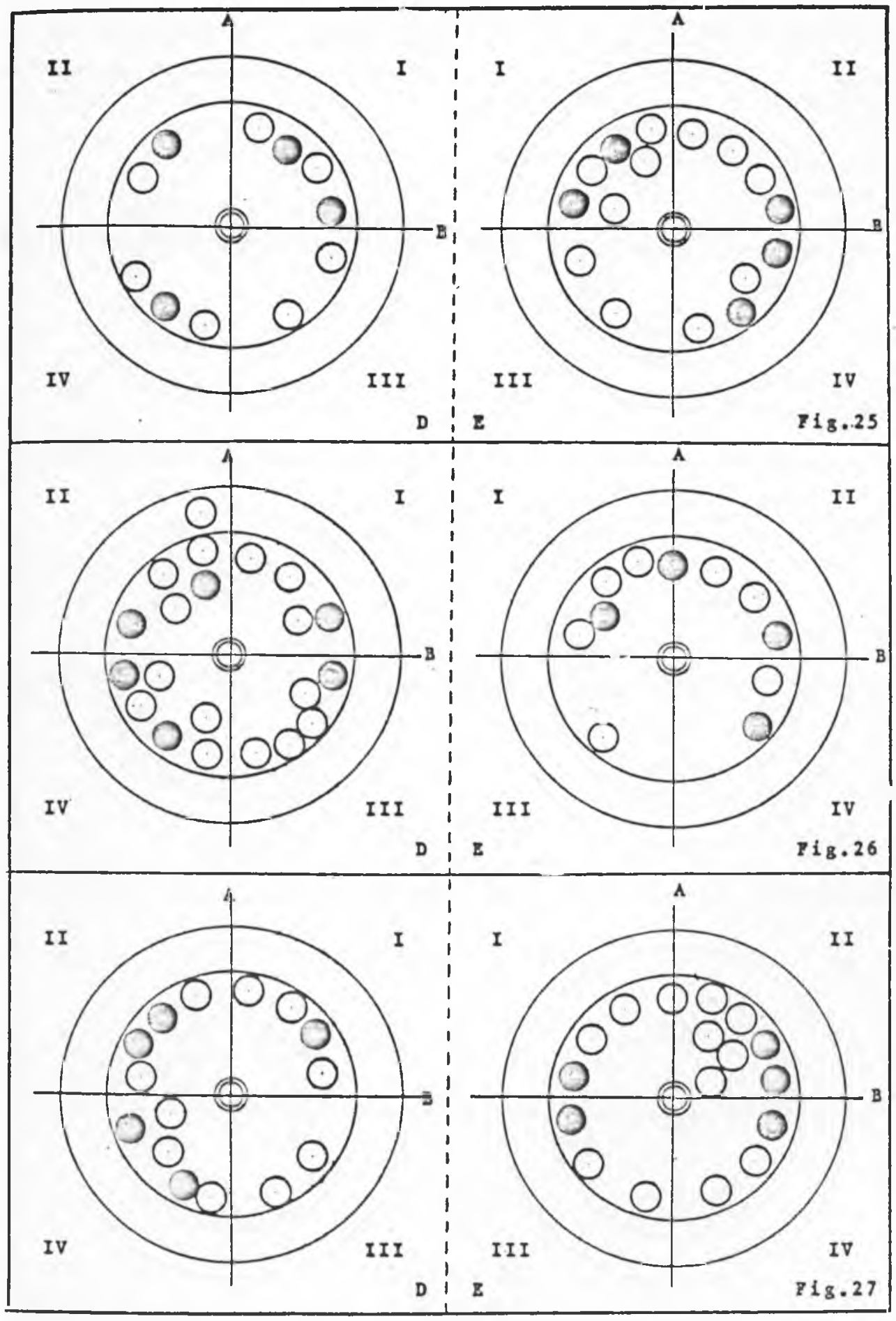

Rev.Fac.Med.vet.Zootec.Univ.S.Paulo, 22(2): 115-33, 1985. 


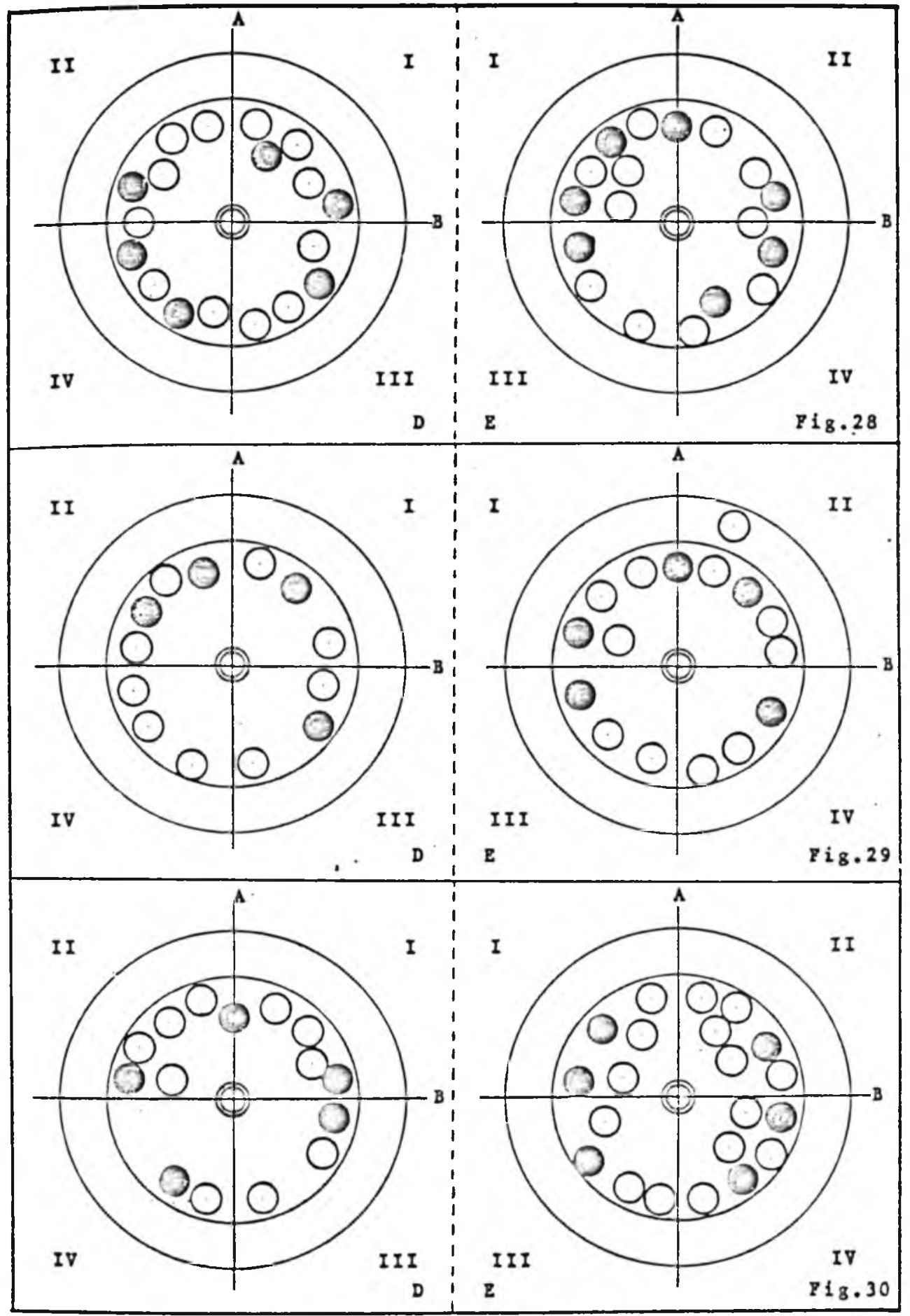

Rev.Fac.Med.vet.Zootec.Univ.S.Paulo, 22(2): 115-33, 1985. 


\section{REFERENCIAS BIBLIOGRĀFICAS}

1- MARTIN, P. \& SCHAUDER, W. Lehrbuch der Anatomie der Haustiere. Stuttgart, Schickhardt \& Ebner, 3(3):277, 1938.

2- MONTANÉ, L. \& BOURDELLE, E. Anatomie régionale des animaux domestiques. Paris, J.B.
Baillière, 1917. v.2.

3- PEDUTI NETO, J. Contribuição ao estudo dos elementos vasculares, arteriais e venosos, do hilo renal em ovinos (Ovis aries, Linnaeus, 1758). São Paulo, 1970. [Tese de mestrado - Faculdade de Medicina Veterinária e Zootecnia da Universidade de São Paulo]

Recebido para publicação em: 16/07/65 Aprovado para publicação em: 30/10/85 